\title{
Optimization of Thiazolidone Scaffolds Using Pocket Modeling for Development of Potential Secretory System Inhibitors of Mycobacterium tuberculosis
}

\section{Mycobacterium tuberculosis'in Potansiyel Sekreter Sistem İnhibitörleri Olarak Thiazolidone İskelelerinin Optimizasyonu}

\author{
(D) Shivratna V. KHARE1, (D) Sujata P. CHOUDHARI2, (D) Siddharth P. PHALLE1, (D) Santosh S. KUMBHAR1, (D) Prafulla B. CHOUDHARI ${ }^{*}$, \\ (D) Sambhaji R. MASAL1, (D) Aakash K. PATIL1, (D) Rakesh P. DHAVALE 3 , (D) Durgacharan A. BHAGWAT3, (D) Atul M. KADAM4
}

1Bharati Vidyapeeth College of Pharmacy, Department of Pharmaceutical Chemistry, Computational Chemistry Research Lab, Kolhapur, India

2Sarojini College of Pharmacy, Department of Pharmaceutical Analysis, Kolhapur, India

3Bharati Vidyapeeth College of Pharmacy, Department of Pharmaceutics, Kolhapur, India

${ }^{4}$ Shree Santkrupa College of Pharmacy, Department of Pharmaceutics, Ghogaon, India

\begin{abstract}
Objectives: Mycobacterium tuberculosis is the causative organism of tuberculosis, which is the most lethal disease after cancer in the current decade. The development of multidrug and broadly drug-resistant strains is making the problem of tuberculosis more and more critical. In the last 40 years, only one molecule has been added to the treatment regimen. Generally, drug design and development programs target proteins whose function is known to be essential to the bacterial cell. M. tuberculosis possesses specialized protein export systems like the SecA2 export pathway and ESX pathways.

Materials and Methods: In the present communication, rational development of an antimycobacterial agent's targeting protein export system was carried out by integrating pocket modeling and virtual analysis.

Results: The 23 identified potential lead compounds were synthesized, characterized by physicochemical and spectroscopic methods like infrared and nuclear magnetic resonance spectroscopy, and further screened for antimycobacterial activity using isoniazid as standard. All the designed compounds showed profound antimycobacterial activity.

Conclusion: We found that Q30, M9, M26, U8, and R26 molecules had significant desirable biological activity and specific interactions with Sec of mycobacteria. Further optimization of these leads is necessary for the development of potential antimycobacterial drug candidates with fewer side effects.
\end{abstract}

Key words: Mycobacterium tuberculosis, Sec, ESX, docking, antimycobacterial, multidrug resistant, pocket modeling

ÖZ

Amaç: Mycobacterium tuberculosis, son on yılda kanserden sonra en ölümcül hastalık olan tüberkülozun etkenidir. Çoklu ilaç ve ilaca dirençli suşların gelișimi, tüberküloz problemini daha da kritik kılmaktadır. Son 40 yılda, tedavi rejimine sadece bir molekül eklenmiștir. Genel olarak ilaç tasarım ve geliştirme programları, bakteri hücresi fonksiyonunda önemi olduğu bilinen proteinleri hedeflemektedir. M. tuberculosis, SecA2 ve ESX gibi özel protein ihracat sistemlerine sahiptir.

Gereç ve Yöntemler: Bu çalıșmada, protein atım sistemini hedefleyen antimikobakteriyel bir bileșiğin rasyonel geliștirilmesi integre cep modelleme ve sanal analiz kullanılarak gerçekleștirilmiştir.

*Correspondence: E-mail: praffula12@gmail.com - prafulla.choudhari@bharatividyapeeth.edu ORCID-ID: orcid.org/0000-0002-9137-3982 Received: 10.02.2018, Accepted: 22.03.2018

๑Turk J Pharm Sci, Published by Galenos Publishing House. 
Bulgular: Yirmi üç bileşik tasarlanmış, sentezlenmiş, fizikokimyasal özellikleri ve infrared ve nükleer manyetik rezonans spektroskopisi ile yapıları karakterize edilmiştir. Ayrıca isoniazid standart olarak kullanılarak antimikobakteriyel etkileri saptanmıştır. Tasarlanan bileșiklerin hepsi iyi antimikobakteriyel aktivite göstermiştir.

Sonuç: Q30, M9, M26, U8 ve R26 moleküllerinin önemli biyolojik aktiviteye ve mikobakterilerin Sec ile spesifik etkileșime sahip olduğu bulunmuştur. Bu hedef bileşiklerin daha da iyileştirilmesi, daha az yan etkiye sahip potansiyel antimikobakteriyel ilaç adaylarının gelişimi için gereklidir.

Anahtar kelimeler: Mycobacterium tuberculosis, Sec, ESX, docking, antimikobakteriyel, çoklu ilaç direnci, cep modellemesi

\section{INTRODUCTION}

Tuberculosis is an air-borne disease caused by infection with Mycobacterium tuberculosis. In the current decade, tuberculosis has emerged as a global emergency due to its mortality rate. ${ }^{1-3}$ Tuberculosis acts as the salient killer in patients suffering from immunocompromising diseases like acquired immune deficiency syndrome (AIDS) and diabetics. In more than $80 \%$ cases of AIDS death occurs in the patients due to tuberculosis. ${ }^{4-7}$ The problem of multidrug resistant tuberculosis (MDR), extensively drug-resistant tuberculosis, and total drug-resistant tuberculosis has reached its peak. ${ }^{8-11}$ Bedaquiline is the only newly developed and approved drug for active MDR tuberculosis in the last two decades. Negligence of pharmaceutical scientists and medicinal chemists towards tuberculosis generated this global problem of tuberculosis. A number of hurdles are normally associated with antitubercular drug discovery; one of them is M. tuberculosis. M. tuberculosis is lipid-rich gram-negative organism having specialized systems that make it different from other microorganisms. ${ }^{12-15}$ Secretory systems are one of the specialized systems present in $M$. tuberculosis, and are key regulators of virulence of $M$. tuberculosis. In M. tuberculosis three different secretory systems, secondary translocase pathway (SEC), twin arginine translocation (TAT), and ESX, are present. ${ }^{16-18}$ The SEC pathway is a major protein export system present in the mycobacterium, accounting for more than $50 \%$ of virulence protein exports in the mycobacterium. The SEC is a conserved protein pathway in the mycobacterium that does not have any homologues in the mammalian systems, and possesses ideal properties to act as a potential antimycobacterial drug target. The SEC pathway is a key enzyme involved in transport of the virulence protein across the cell membrane, which spreads tuberculosis all over the host body. Inhibition of SEC will be able to block the transport of the virulence protein, which will inhibit the spread of the tuberculosis. In recent years, a number of researchers have been trying to develop novel antitubercular agents targeting conserved protein targets. Currently a number of molecules like equisetin derivatives ${ }^{19}$ and thiazolo[4,5-d] pyrimidine derivatives ${ }^{20}$ are reported as SEC inhibitors. In this research article, an attempt was made to optimize thiazolidine scaffolds as potential SEC inhibitors via integration of pocket and pharmacophore modeling.

\section{EXPERIMENTAL}

\section{Selection of target}

M. tuberculosis has three specialized protein export systems: SEC, TAT, and ESX. The SEC is major protein export system of $M$. tuberculosis, exporting the bulk of virulent protein, which accounts for the spread of disease. SEC systems do not have any homologues in mammalian systems and so inhibition of this SEC will not lead to any toxicity to humans. Thus due to its critical role in the growth and virulence of the M. tuberculosis, the SEC has been selected as the preferred biomolecular target rather than TAT or ESX.

\section{Pocket modeling of the selected protein export systems}

Pocket modeling of the selected SEC of M. tuberculosis was carried out using the crystal structure of SEC downloaded from the free protein database www.rcsb.org. The downloaded crystal structure of SEC was first refined by the BioPredicta module, via removal of water molecules and retaining native hydrogen atoms in the protein structure. Pocket modeling of SEC A was carried out using the ProViz module of Vlife MDS 4.4. ProViz is an integrated property visualization module with which electrostatic and hydrophobic mapping of biomolecules can be carried out.

\section{Design of molecules}

Pocket modeling of the SEC revealed the binding pocket of SEC is U-shaped and highly hydrophobic, keeping in mind complementary structures on the thiazole template were designed with structural modification on the aromatic moiety carried out. A number of the aromatic benzaldehydes are utilized to generate a number of thiazole derivatives. Total $75 \times 75$ benzaldehyde combinations are utilized to generate total 5625 different structures (indicated as R) as shown in Table 1. These 5625 different thiazole derivatives were designed and drawn in 2D geometry using the builder module of Vlife MDS 4.4. 2D structures were converted into 3D geometry and their coordinates were optimized via energy minimization by application of Merck molecular force field.

\section{Docking analysis of synthesized ligands}

Molecular docking was performed to identify potent derivatives with the maximum binding affinity for SEC of M. tuberculosis amongst the designed set of molecules. Docking analysis was performed in the BioPredicta module of Vlife MDS 4.4 using grip-based docking analysis in which the protein structure was kept rigid and molecules were kept in flexible conformation so that a number of conformations can be achieved. The best 100 confirmations of all designed molecules were generated. Potent molecules were scrutinized based on binding energy and interaction profile. The proposed synthetic scheme of the designed derivatives is shown in Figure 1.

Screening based on the drug-like properties and percentage absorption

The designed set of molecules was analyzed for Lipinski parameters like molecular weight, $\mathrm{H}$-bond acceptor, $\mathrm{H}$-bond 
Table 1. Designed set of molecules (total $75 \times 75$ benzaldehyde combinations are utilized to generate total 5625 different structures indicated as R)

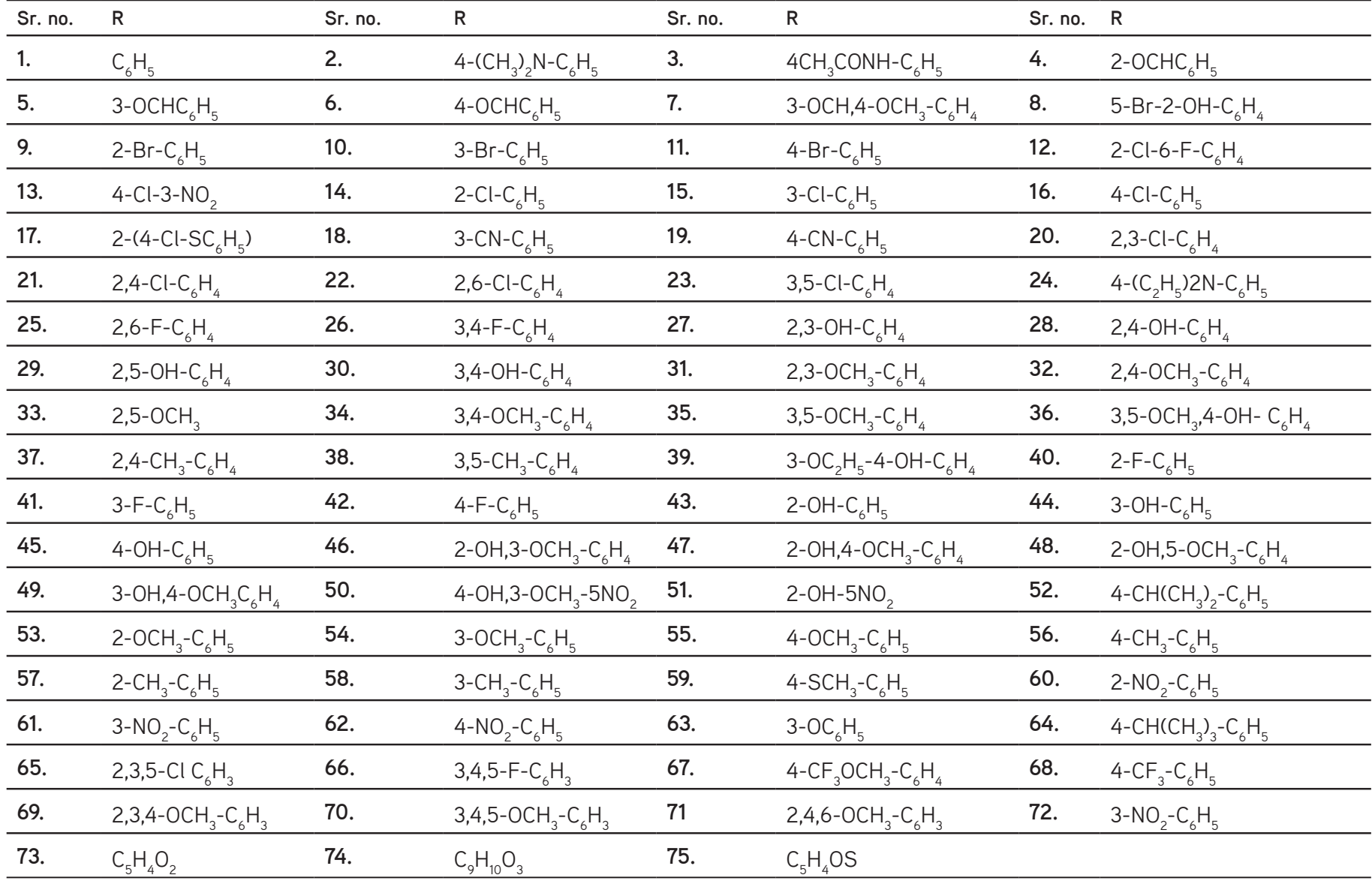

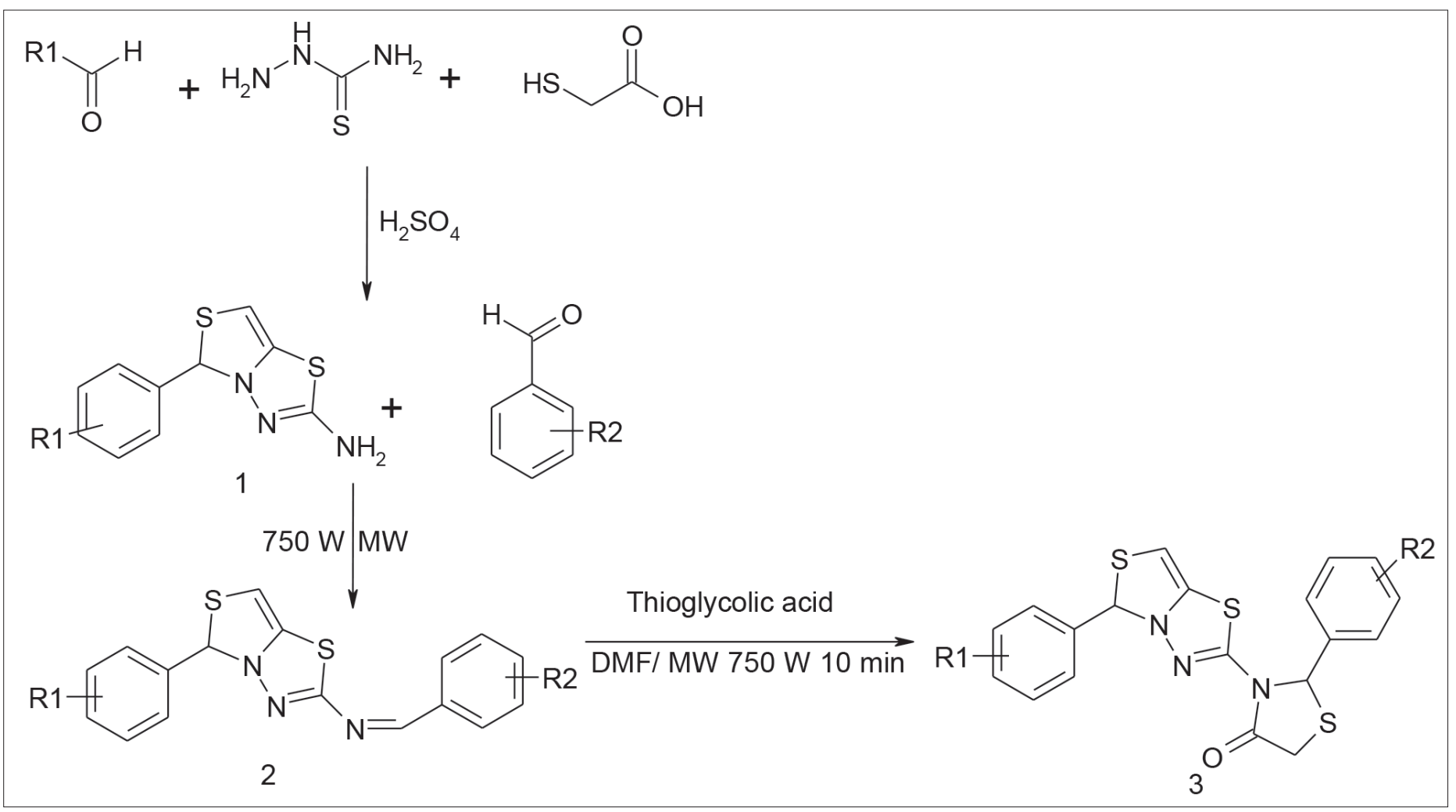

Figure 1. Synthetic scheme of designed derivatives 
donor, rotatable bond, and XlogP. The physicochemical descriptors were calculated with the help of the QSAR module of Vlife MDS 4.4. Total polar surface area (TPSA) was calculated from the web server www.molinspiration.com/cgibin/properties by drawing the molecules in the drawing area and then calculating the TPSA. Percentage absorption was calculated using the formula;

$\%$ Absorption = $109-(0.345 \times$ TPSA $){ }^{21}$

Synthesis of selected ligands ${ }^{22}$

Synthesis of optimized thiazole derivatives

Step 1

Synthesis of 2-amino-5-aryl-5H thiazolo[4,3-b]-l,3,4thiadiazole (1)

To $25 \mathrm{~mL}$ of RBF were added aldehyde (0.02 M), thioglycolic acid $(0.02 \mathrm{M})$, thiosemicarbazide $(0.022 \mathrm{M})$, and $10 \mathrm{~mL}$ of concentrated $\mathrm{H}_{2} \mathrm{SO}_{4}$. The reaction mixture was mixed and left overnight and the resulting suspension was neutralized with $40 \% \mathrm{NaOH}$ solution until the product was precipitated out and this resulting compound 1 was recrystallized from aqueous dioxane solution.

Synthesis of 1-phenyl-N-\{5-phenyl-5H-[1,3]thiazolo[4,3-b] [1,3,4] thiadiazol-2-yl\}methanimine derivatives (2):

A solution of compound $1(0.01 \mathrm{M})$ in ethanol $(50 \mathrm{~mL})$ was put in RBF and stirred vigorously for $15 \mathrm{~min}$ and to this resulting solution concentrated $\mathrm{H}_{2} \mathrm{SO}_{4}(2 \mathrm{~mL})$ and aldehyde $(0.01 \mathrm{M})$ were added. The reaction mixture was irradiated in a synthetic microwave (Cata 4R) for $10 \mathrm{~min}$. The separated solid was filtered and recrystallized from ethanol.

Synthesis of 2-phenyl-3-\{5-phenyl-5H-[1,3]thiazolo[4,3-b] [1,3,4] thiadiazol-2-yl\}-1,3- thiazolidin-4-one derivatives (3):

In $25 \mathrm{~mL}$ of RBF were added compound 2 (0.01 M) and thioglycolic acid $(0.01 \mathrm{M})$ and then $30 \mathrm{~mL}$ of DMF was added followed by stirring. The resulting reaction mixture was irradiated in the microwave for $10 \mathrm{~min}$ at $750 \mathrm{~W}$. The reaction mixture was cooled to room temperature and the resulting solid was separated and recrystallized from benzene to get compounds 1 to 23. Table 2 shows the list of substituents in the synthesized set of molecules.

B3: $\quad 3-[5-(4-b r o m o p h e n y l)-5 H-[1,3]$ thiazolo[4,3-b][1,3,4] thiadiazol-2-yl]-2-phenyl-1,3 thiazolidin-4-one

Color: Brown, Yield: 82\%, m. p.: 192-194 ${ }^{\circ} \mathrm{C}$, MASS: [M+1] + 474.94, IR: $1752 \mathrm{~cm}^{-1}$ (C=O Str.), $1452 \mathrm{~cm}^{-1}(\mathrm{C}=\mathrm{C}), 2742.84 \mathrm{~cm}^{-1}(\mathrm{Ar} . \mathrm{CH})$, $1580 \mathrm{~cm}^{-1}(-\mathrm{N}=\mathrm{CH}) \mathrm{NMR}:{ }^{1} \mathrm{H}$ NMR (DMSO-d, $300 \mathrm{MHz}$ ): $\delta=6.95-$ 7.35 (m, 9H, aromatic H), 4.95-5.90 (s, 2H, methine), 3.35 (s, $2 \mathrm{H}$, methylene), 4.85 (s, $1 \mathrm{H}$, ethylene).

C13: 2-(4-chlorophenyl)-3-\{5-phenyl-5H-[1,3]thiazolo[4,3-b] [1,3,4]thiadiazol-2-yl\}-1,3-thiazolidin-4-one

Color: Yellow, Yield: $80 \%$, m. p.: $178-180^{\circ} \mathrm{C}$, MASS: $[\mathrm{M}+1]+$ 430.99, IR: $1690 \mathrm{~cm}^{-1}$ (C=O Str.), $1435 \mathrm{~cm}^{-1}(\mathrm{C}=\mathrm{C}), 2842 \mathrm{~cm}^{-1}(\mathrm{Ar}$. $\mathrm{CH}), 1470 \mathrm{~cm}^{-1}(-\mathrm{N}=\mathrm{CH}), \mathrm{NMR}:{ }^{1} \mathrm{H}$ NMR (DMSO-d, $300 \mathrm{MHz}$ ): $\delta=6.90-7.25$ (m, 9H, aromatic), 4.95-5.90 (s, 2H, methine), 3.38 (s, $2 \mathrm{H}$, methylene), 4.95 (s, $1 \mathrm{H}$, ethylene).
C1: 2-(2-chlorophenyl)-3-\{5-phenyl-5H-[1,3]thiazolo[4,3-b] [1,3,4]thiadiazol-2-yl\}-1,3-thiazolidin-4-one

Color: Brown, Yield: $75 \%$, m. p.: $186-188^{\circ} \mathrm{C}$, MASS: $[\mathrm{M}+1]+$ 430.99, IR: $1625 \mathrm{~cm}^{-1}$ (C=O Str.), $1400 \mathrm{~cm}^{-1}(\mathrm{C}=\mathrm{C}), 2830 \mathrm{~cm}^{-1}$ (Ar. $\mathrm{CH}), 1476 \mathrm{~cm}^{-1}(-\mathrm{N}=\mathrm{CH})$ NMR: ${ }^{1} \mathrm{H}$ NMR (DMSO- $d_{6}, 300 \mathrm{MHz}$,): $\delta=6.95-7.20(\mathrm{~m}, 9 \mathrm{H}$, aromatic), 4.90-5.92 (s, 2H, methine), 3.30 (s, $2 \mathrm{H}$, methylene), 4.90 (s, $1 \mathrm{H}$, ethylene).

F1: 2-(2-chlorophenyl)-3-[5-(3,4-dimethoxyphenyl)-5H-[1,3] thiazolo[4,3-b][1,3,4]thiadiazol-2-yl]-1,3-thiazolidin-4-one Color: Lemon, Yield: $89 \%$, m. p.: $162-164^{\circ} \mathrm{C}$, MASS: [M+1] + 492.02, IR: $1640 \mathrm{~cm}^{-1}$ (C=O Str.), $1490 \mathrm{~cm}^{-1}(\mathrm{C}=\mathrm{C}), 2790 \mathrm{~cm}^{-1}$ (Ar. $\mathrm{CH}$ ), $1520 \mathrm{~cm}^{-1}\left(-\mathrm{N}=\mathrm{CH}\right.$ ) NMR: ${ }^{1} \mathrm{H}$ NMR (DMSO-d ${ }_{6}, 300 \mathrm{MHz}$,): $\delta=6.52-7.15(\mathrm{~m}, 7 \mathrm{H}$, aromatic), 4.95-5.90 (s, $2 \mathrm{H}$, methine), 3.33 (s, $2 \mathrm{H}$, methylene), 4.85 (s, $1 \mathrm{H}$, ethylene), 3.75 (d, 6H, methyl).

017: 2-(3-chlorophenyl)-3-[5-(2-hydroxyphenyl)-5H-[1,3] thiazolo[4,3-b][1,3,4] thiadiazol-2-yl]-1,3-thiazolidin-4-one Color: Yellow, Yield: $80 \%$, m. p.: $228-230^{\circ} \mathrm{C}$, MASS: [M+1] + 447.97, IR: $1680 \mathrm{~cm}^{-1}$ (C=O Str.), $1500 \mathrm{~cm}^{-1}(\mathrm{C}=\mathrm{C}), 2810 \mathrm{~cm}^{-1}$ (Ar. $\mathrm{CH}), 1580 \mathrm{~cm}^{-1}\left(-\mathrm{N}=\mathrm{CH}\right.$ ) NMR: ${ }^{1} \mathrm{H}$ NMR (DMSO- $d_{6}, 300 \mathrm{MHz}$,):

Table 2. List of substituents in synthesized set of molecules

\begin{tabular}{|c|c|c|c|}
\hline Sr. no. & $\mathrm{R} 1$ & $\mathrm{R} 2$ & Code \\
\hline 1. & $4-\mathrm{Br}-\mathrm{C}_{6} \mathrm{H}_{4}$ & $-\mathrm{C}_{6} \mathrm{H}_{4}$ & B3 \\
\hline 2. & $-\mathrm{C}_{6} \mathrm{H}_{4}$ & $4-\mathrm{Cl}-\mathrm{C}_{6} \mathrm{H}_{4}$ & $\mathrm{C} 13$ \\
\hline 3. & $-\mathrm{C}_{6} \mathrm{H}_{4}$ & $2-\mathrm{Cl}-\mathrm{C}_{6} \mathrm{H}_{4}$ & $\mathrm{C} 1$ \\
\hline 4. & $3,4-\left(\mathrm{OCH}_{3}\right)-\mathrm{C}_{6} \mathrm{H}_{4}$ & $2-\mathrm{Cl}-\mathrm{C}_{6} \mathrm{H}_{4}$ & $\mathrm{~F} 1$ \\
\hline 5. & $2-(\mathrm{OH})-\mathrm{C}_{6} \mathrm{H}_{4}$ & $3-\mathrm{Cl}-\mathrm{C}_{6} \mathrm{H}_{4}$ & 017 \\
\hline 6. & $-\mathrm{C}_{4} \mathrm{H}_{3} \mathrm{O}$ & $3-\mathrm{OCH}_{3}-4-\mathrm{OH}-\mathrm{C}_{6} \mathrm{H}_{3}$ & Q21 \\
\hline 7. & $4-\mathrm{N}\left(\mathrm{CH}_{3}\right)_{2}-\mathrm{C}_{6} \mathrm{H}_{4}$ & $4-\mathrm{Cl}-\mathrm{C}_{6} \mathrm{H}_{4}$ & L13 \\
\hline 8. & $2-(\mathrm{OH})-\mathrm{C}_{6} \mathrm{H}_{4}$ & $4-\mathrm{Cl}-\mathrm{C}_{6} \mathrm{H}_{4}$ & 013 \\
\hline 9. & $-\mathrm{C}_{4} \mathrm{H}_{3} \mathrm{O}$ & $2-(\mathrm{OH})-\mathrm{C}_{6} \mathrm{H}_{4}$ & Q24 \\
\hline 10. & $4-\mathrm{Cl}-\mathrm{C}_{6} \mathrm{H}_{4}$ & 4- $(\mathrm{OH})-\mathrm{C}_{6} \mathrm{H}_{4}$ & M9 \\
\hline 11. & $4-\mathrm{F}-\mathrm{C}_{6} \mathrm{H}_{4}$ & $-\mathrm{C}_{4} \mathrm{H}_{3} \mathrm{O}$ & $\mathrm{H} 25$ \\
\hline 12. & $3-\mathrm{Cl}-\mathrm{C}_{6} \mathrm{H}_{4}$ & $\mathrm{~S}-\mathrm{C}_{4} \mathrm{H}_{3}$ & R26 \\
\hline 13. & 3- $\left(\mathrm{OCH}_{3}\right)-4-(\mathrm{OH})-\mathrm{C}_{6} \mathrm{H}_{3}$ & $-\mathrm{C}_{6} \mathrm{H}_{4}$ & V3 \\
\hline 14. & $3,5-\left(\mathrm{OCH}_{3}\right)_{2}-4-(\mathrm{OH}) \mathrm{C}_{6} \mathrm{H}_{2}$ & $-\mathrm{C}_{6} \mathrm{H}_{4}$ & U8 \\
\hline 15. & $3-\mathrm{Cl}-\mathrm{C}_{6} \mathrm{H}_{4}$ & $3-\mathrm{NO}_{2}-\mathrm{C}_{6} \mathrm{H}_{4}$ & A11 \\
\hline 16. & $2-\mathrm{Cl}-\mathrm{C}_{6} \mathrm{H}_{4}$ & $4-\mathrm{NO}_{2}-\mathrm{C}_{6} \mathrm{H}_{4}$ & A10 \\
\hline 17. & $-\mathrm{C}_{4} \mathrm{H}_{3} \mathrm{O}$ & $3-\mathrm{OCH}_{3}-\mathrm{C}_{6} \mathrm{H}_{4}$ & Q22 \\
\hline 18. & $-\mathrm{C}_{4} \mathrm{H}_{3} \mathrm{O}$ & $-\mathrm{C}_{7} \mathrm{H}_{5} \mathrm{O}_{2}$ & Q30 \\
\hline 19. & $3-\mathrm{Cl}-\mathrm{C}_{6} \mathrm{H}_{4}$ & $\mathrm{~S}-\mathrm{C}_{4} \mathrm{H}_{3}$ & R25 \\
\hline 20. & $4-\mathrm{Cl}-\mathrm{C}_{6} \mathrm{H}_{4}$ & $\mathrm{~S}-\mathrm{C}_{4} \mathrm{H}_{3}$ & M26 \\
\hline 21. & $3-\mathrm{Cl}-\mathrm{C}_{6} \mathrm{H}_{4}$ & $3,4-\left(\mathrm{OCH}_{3}\right)-\mathrm{C}_{6} \mathrm{H}_{4}$ & R6 \\
\hline 22. & 3- $\left(\mathrm{OCH}_{3}\right)-\mathrm{C}_{6} \mathrm{H}_{4}$ & 4- $\mathrm{Cl}-\mathrm{C}_{6} \mathrm{H}_{4}$ & W13 \\
\hline 23. & 3- $\left(\mathrm{OCH}_{3}\right)-\mathrm{C}_{6} \mathrm{H}_{4}$ & $4-\mathrm{F}-\mathrm{C}_{6} \mathrm{H}_{4}$ & w8 \\
\hline
\end{tabular}


$\delta=6.62-7.20(\mathrm{~m}, 8 \mathrm{H}$, aromatic), 4.95 (s, $1 \mathrm{H}$, methine), $5.92(\mathrm{~s}, 1 \mathrm{H}$, methine), 3.38 (s, $1 \mathrm{H}$, methylene), 4.75 (s, $1 \mathrm{H}$, ethylene), 6.05 (s, $1 \mathrm{H}$, aromatic $\mathrm{C}-\mathrm{OH})$.

Q21: $\quad$ 2-(3-ethoxy-4-hydroxyphenyl)-3-[5-(furan-2-yl)$5 \mathrm{H}$-[1,3]thiazolo[4,3-b][1,3,4] thiadiazol-2-yl]-1,3-thiazolidin-4one

Color: Black, Yield: $78 \%$, m. p.: $192-194^{\circ} \mathrm{C}$, MASS: [M+1] + 447.54, IR: $1750 \mathrm{~cm}^{-1}$ (C=O Str.), $1540 \mathrm{~cm}^{-1}(\mathrm{C}=\mathrm{C}), 2840 \mathrm{~cm}^{-1}$ (Ar. CH), $1600 \mathrm{~cm}^{-1}(-\mathrm{N}=\mathrm{CH}) \mathrm{NMR}:{ }^{1} \mathrm{H}$ NMR (DMSO-d, $300 \mathrm{MHz}$ ) $: \delta=6.05-$ $7.30(\mathrm{~m}, 6 \mathrm{H}$, aromatic benzene and furan), 4.75 (s, $1 \mathrm{H}$, ethylene), 5.15-5.92 (s, 2H, methine), 3.30 (s, $2 \mathrm{H}$, methylene), 3.95 (s, $2 \mathrm{H}$, methylene), 1.58 (s, 3H, methyl), 5.02 (s, $1 \mathrm{H}$ aromatic $\mathrm{C}-\mathrm{OH}$ ).

L13: 2-(4-chlorophenyl)-3-\{5-[4-(dimethylamino)phenyl]$5 \mathrm{H}$-[1,3] thiazolo[4,3-b][1,3,4] thiadiazol-2-yl\}-1,3-thiazolidin-4one

Color: Brown, Yield: $67 \%$, m. p.: $156-158^{\circ} \mathrm{C}$, MASS: $[\mathrm{M}+1]+$ 475.04, IR: $1652 \mathrm{~cm}^{-1}$ (C=O Str.), $1538 \mathrm{~cm}^{-1}(\mathrm{C}=\mathrm{C}), 2800 \mathrm{~cm}^{-1}$ (Ar. $\mathrm{CH}), 1530 \mathrm{~cm}^{-1}(-\mathrm{N}=\mathrm{CH}) \mathrm{NMR}:{ }^{1} \mathrm{H}$ NMR (DMSO- $d_{6}, 300 \mathrm{MHz}$,): $\delta=6.45-7.20(\mathrm{~m}, 8 \mathrm{H}$, aromatic benzene), 4.80 ( $\mathrm{s}, 1 \mathrm{H}$, ethylene), 4.95-5.80 (s, 2H, methine), 3.30 (s, $2 \mathrm{H}$, methylene), 2.95 (s, $6 \mathrm{H}$, methyl $\mathrm{N}-\mathrm{CH}_{3}$ ).

013: 2-(4-chlorophenyl)-3-[5-(2-hydroxyphenyl)-5H-[1,3] thiazolo[4,3-b][1,3,4]thiadiazol-2-yl]-1,3-thiazolidin-4-one

Color: Brown, Yield: 67\%, m. p.: $236-238^{\circ} \mathrm{C}$, MASS: $[\mathrm{M}+1]+$ 447.97, IR: $1760 \mathrm{~cm}^{-1}$ (C=O Str.), $1540 \mathrm{~cm}^{-1}(\mathrm{C}=\mathrm{C}), 2830 \mathrm{~cm}^{-1}$ (Ar. $\mathrm{CH}), 1680 \mathrm{~cm}^{-1}(-\mathrm{N}=\mathrm{CH})$ NMR: ${ }^{1} \mathrm{H}$ NMR (DMSO-d, $300 \mathrm{MHz}$,): $\delta=6.65-7.25(\mathrm{~m}, 8 \mathrm{H}$, aromatic benzene), 4.85 (s, $1 \mathrm{H}$, ethylene), 4.05-4.70 (s, 2H, methine), 3.28 (s, $2 \mathrm{H}$, methylene), 5.25 (s, $1 \mathrm{H}$, aromatic $\mathrm{C}-\mathrm{OH})$.

Q24: $\quad 3-[5-($ furan-2-yl)-5H-[1,3]thiazolo[4,3-b][1,3,4] thiadiazol-2-yl]-2-(2-hydroxyphenyl)-1,3-thiazolidin-4-one Color: Black, Yield: $75 \%$, m. p.: $216-218^{\circ} \mathrm{C}$, MASS: $[\mathrm{M}+1]+$ 403.49, IR: $1740 \mathrm{~cm}^{-1}$ (C=O Str.), $1623 \mathrm{~cm}^{-1}$ (C=C), $2810 \mathrm{~cm}^{-1}$ (Ar. $\mathrm{CH}), 1680 \mathrm{~cm}^{-1}(-\mathrm{N}=\mathrm{CH}), \mathrm{NMR}:{ }^{1} \mathrm{H}$ NMR (DMSO-d, $300 \mathrm{MHz}$,): $\delta=6.60-7.30(\mathrm{~m}, 7 \mathrm{H}$, aromatic benzene and furan), $4.80(\mathrm{~s}, 1 \mathrm{H}$, ethylene), 5.20-5.80 (s, 2H, methine), 3.30 (s, $2 \mathrm{H}$, methylene), 5.25 (s, $1 \mathrm{H}$, aromatic $\mathrm{C}-\mathrm{OH})$.

M9: 3-[5-(4-chlorophenyl)-5H-[1,3]thiazolo[4,3-b][1,3,4] thiadiazol-2-yl]-2-(4-hydroxyphenyl)-1,3-thiazolidin-4-one Color: Brown, Yield: $88 \%$, m. p.: $138-140^{\circ} \mathrm{C}$, MASS: $[\mathrm{M}+1]+$ 447.97, IR: $1700 \mathrm{~cm}^{-1}$ (C=O Str.), $1640 \mathrm{~cm}^{-1}$ (C=C), $2790 \mathrm{~cm}^{-1}$ (Ar. $\mathrm{CH}), 1650 \mathrm{~cm}^{-1}(-\mathrm{N}=\mathrm{CH})$, NMR: ${ }^{1} \mathrm{H}$ NMR (DMSO-d, $300 \mathrm{MHz}$,): $\delta=6.55-7.25(\mathrm{~m}, 8 \mathrm{H}$, aromatic benzene), 4.85 (s, $1 \mathrm{H}$, ethylene), 5.10-5.85 (s, 2H, methine), 3.38 (s, 2H, methylene), 6.05 (s, $1 \mathrm{H}$, aromatic $\mathrm{C}-\mathrm{OH})$.

H25: 3-[5-(4-fluorophenyl)-5H-[1,3]thiazolo[4,3-b][1,3,4] thiadiazol-2-yl]-2-(furan-2-yl)-1,3-thiazolidin-4-one

Color: Yellow, Yield: $70 \%$, m. p.: $122-124^{\circ} \mathrm{C}$, MASS: $[\mathrm{M}+1]+$ 405.48, IR: $1688 \mathrm{~cm}^{-1}$ (C=O Str.), $1589 \mathrm{~cm}^{-1}(\mathrm{C}=\mathrm{C}), 2690 \mathrm{~cm}^{-1}$ (Ar. $\mathrm{CH}), 1580 \mathrm{~cm}^{-1}(-\mathrm{N}=\mathrm{CH})$ NMR: ${ }^{1} \mathrm{H}$ NMR (DMSO-d, $300 \mathrm{MHz}$,): $\delta=6.30-7.05(\mathrm{~m}, 7 \mathrm{H}$, aromatic benzene and furan), $4.82(\mathrm{~s}, 1 \mathrm{H}$, ethylene), 5.10-5.85 (s, 2H, methine), 3.35 (s, 2H, methylene).

R26: 3-[5-(3-chlorophenyl)-5H-[1,3]thiazolo[4,3-b][1,3,4] thiadiazol-2-yl]-2-(thiophen-2-yl)-1,3-thiazolidin-4-one

Color: Yellow, Yield: $72 \%$, m. p.: $206-208^{\circ} \mathrm{C}$, MASS: $[\mathrm{M}+1]+$ 437.99, IR: $1750 \mathrm{~cm}^{-1}$ (C=O Str.), $1670 \mathrm{~cm}^{-1}(\mathrm{C}=\mathrm{C}), 2560 \mathrm{~cm}^{-1}$ (Ar. CH), $1540 \mathrm{~cm}^{-1}(-\mathrm{N}=\mathrm{CH})$ NMR: ${ }^{1} \mathrm{H}$ NMR (DMSO-d, 300 $\mathrm{MHz}$ ): $\delta=6.60-7.10(\mathrm{~m}, 7 \mathrm{H}$, aromatic benzene and thiophene), 4.85 (s, $1 \mathrm{H}$, ethylene), 4.95-5.90 (s, $2 \mathrm{H}$, methine), 3.30 (s, $2 \mathrm{H}$, methylene).

V3: 3-[5-(3-ethoxy-4-hydroxyphenyl)-5H-[1,3]thiazolo[4,3-b] [1,3,4]thiadiazol-2-yl]-2-phenyl-1,3-thiazolidin-4-one

Color: Brown, Yield: $83 \%$, m. p.: $180-182^{\circ} \mathrm{C}$, MASS: $[\mathrm{M}+1]+$ 457.58, IR: $1620 \mathrm{~cm}^{-1}$ (C=O Str.), $1560 \mathrm{~cm}^{-1}$ (C=C), $2860 \mathrm{~cm}^{-1}$ (Ar. $\mathrm{CH}), 1490 \mathrm{~cm}^{-1}(-\mathrm{N}=\mathrm{CH})$ NMR: ${ }^{1} \mathrm{H}$ NMR (DMSO-d, $300 \mathrm{MHz}$,): $\delta=6.45-7.14(\mathrm{~m}, 8 \mathrm{H}$, aromatic benzene), $4.80(\mathrm{~s}, 1 \mathrm{H}$, ethylene), 4.90-5.85 (s, 2H, methine), 3.35 (s, 2H, methylene), 5.95 (s, $1 \mathrm{H}$, aromatic $\mathrm{C}-\mathrm{OH}$ ), 3.98 (s, $2 \mathrm{H}$, methylene), 1.75 (s, 3H, methyl).

U8: 3-[5-(4-hydroxy-3,5-dimethoxyphenyl)-5H-[1,3] thiazolo[4,3-b][1,3,4]thiadiazol-2-yl]-2-phenyl-1,3-thiazolidin4-one

Color: Yellow, Yield: $80 \%$, m. p.: $230-232^{\circ} \mathrm{C}$, MASS: $[\mathrm{M}+1]+$ 473.58, IR: $1688 \mathrm{~cm}^{-1}$ (C=O Str.), $1562 \mathrm{~cm}^{-1}(\mathrm{C}=\mathrm{C}), 2883 \mathrm{~cm}^{-1}$ (Ar. $\mathrm{CH}), 1489 \mathrm{~cm}^{-1}(-\mathrm{N}=\mathrm{CH})$ NMR: ${ }^{1} \mathrm{H}$ NMR (DMSO-d, $300 \mathrm{MHz}$,): $\delta=5.95-7.10(\mathrm{~m}, 7 \mathrm{H}$, aromatic benzene), 4.85 (s, $1 \mathrm{H}$, ethylene), 4.92-5.95 (s, 2H, methine), 3.28 (s, $2 \mathrm{H}$, methylene), 5.98 (s, $1 \mathrm{H}$, aromatic $\mathrm{C}-\mathrm{OH}$ ), 3.85 (s, $6 \mathrm{H}$, methoxy).

A11: $\quad 3-[5-(3-c h l o r o p h e n y l)-5 H-[1,3]$ thiazolo[4,3-b][1,3,4] thiadiazol-2-yl]-2-(3-nitrophenyl)-1,3-thiazolidin-4-one

Color: Brown, Yield: $69 \%$, m. p.: $146-148^{\circ} \mathrm{C}$, MASS: $[\mathrm{M}+1]+$ 476.97, IR: $1679 \mathrm{~cm}^{-1}$ (C=O Str.), $1575 \mathrm{~cm}^{-1}(\mathrm{C}=\mathrm{C}), 2896 \mathrm{~cm}^{-1}$ (Ar. $\mathrm{CH}), 1523 \mathrm{~cm}^{-1}(-\mathrm{N}=\mathrm{CH})$. NMR: ${ }^{1} \mathrm{H}$ NMR (DMSO-d, $300 \mathrm{MHz}$,): $\delta=6.90-7.95(\mathrm{~m}, 8 \mathrm{H}$, aromatic benzene), 4.75 (s, 1H, ethylene), 4.90-5.92 (s, 2H, methine), 3.33 (s, 2H, methylene).

A10: $\quad 3-[5-(2-c h l o r o p h e n y l)-5 H-[1,3]$ thiazolo[4,3-b][1,3,4] thiadiazol-2-yl]-2-(4-nitrophenyl)-1,3-thiazolidin-4-one Color: Brown, Yield: $70 \%$, m. p.: $174-176^{\circ} \mathrm{C}$, MASS: $[\mathrm{M}+1]+$ 476.97, IR: $1590 \mathrm{~cm}^{-1}$ (C=O Str.), $1456 \mathrm{~cm}^{-1}\left(\mathrm{C}=\mathrm{C}\right.$ ), $2675 \mathrm{~cm}^{-1}$ (Ar. $\mathrm{CH}), 1563 \mathrm{~cm}^{-1}(-\mathrm{N}=\mathrm{CH})$. NMR: ${ }^{1} \mathrm{H}$ NMR (DMSO-d, $300 \mathrm{MHz}$,): $\delta=7.04-8.05(\mathrm{~m}, 8 \mathrm{H}$, aromatic benzene), 4.85 ( $\mathrm{s}, 1 \mathrm{H}$, ethylene), 4.95-5.90 (s, 2H, methine), 3.28 (s, 2H, methylene).

Q22: $\quad 3-[5-(f u r a n-2-y l)-5 H-[1,3]$ thiazolo[4,3-b][1,3,4] thiadiazol-2-yl]-2-(3-methoxyphenyl)-1,3-thiazolidin-4-one Color: Black, Yield: $78 \%$, m. p.: $176-178^{\circ} \mathrm{C}$, MASS: [M+1] + 417.52, IR: $1639 \mathrm{~cm}^{-1}\left(\mathrm{C}=\mathrm{O}\right.$ Str.), $1420 \mathrm{~cm}^{-1}(\mathrm{C}=\mathrm{C}), 2640 \mathrm{~cm}^{-1}(\mathrm{Ar} . \mathrm{CH})$, $1570 \mathrm{~cm}^{-1}(-\mathrm{N}=\mathrm{CH})$. NMR: 'H NMR (DMSO- ${ }_{6}, 300 \mathrm{MHz}$,): $\delta=6.50-$ $7.30(\mathrm{~m}, 7 \mathrm{H}$, aromatic benzene and furan), 4.80 (s, $1 \mathrm{H}$, ethylene), 5.10-5.95 (s, 2H, methine), 3.30 (s, 2H, methylene), 3.75 (s, 3H, methoxy).

Q30: 2-(2H-1,3-benzodioxol-5-yl)-3-[5-(furan-2-yl)-5H-[1,3] thiazolo[4,3-b][1,3,4]thiadiazol-2-yl]-1,3-thiazolidin-4-one 
Color: Black, Yield: $70 \%$, m. p.: $240-242^{\circ} \mathrm{C}$, MASS: [M+1] + 431.50, IR: $1782 \mathrm{~cm}^{-1}$ (C=O Str.), $1560 \mathrm{~cm}^{-1}(\mathrm{C}=\mathrm{C}), 2785 \mathrm{~cm}^{-1}$ (Ar. $\mathrm{CH}), 1630 \mathrm{~cm}^{-1}(-\mathrm{N}=\mathrm{CH})$. NMR: ${ }^{1} \mathrm{H}$ NMR (DMSO- $d_{6}, 300 \mathrm{MHz}$ ): $\delta=6.45-7.25(\mathrm{~m}, 6 \mathrm{H}$, aromatic benzene and furan), $4.82(\mathrm{~s}, 1 \mathrm{H}$, ethylene), 5.15-5.85 (s, 2H, methine), 3.28 (s, $2 \mathrm{H}$, methylene), 5.95 (s, 2H, 1,3-dioxole).

\section{R25: $\quad 3-[5-(3-c h l o r o p h e n y l)-5 H-[1,3]$ thiazolo[4,3-b][1,3,4]} thiadiazol-2-yl]-2-(thiophen-2-yl)-1,3-thiazolidin-4-one Color: Yellow, Yield: $82 \%$, m. p.: $234-236^{\circ} \mathrm{C}$, MASS: $[\mathrm{M}+1]+$ 437.99 IR: $1700 \mathrm{~cm}^{-1}$ (C=O Str.), $1590 \mathrm{~cm}^{-1}$ (C=C), $2693 \mathrm{~cm}^{-1}$ (Ar. CH), $1578 \mathrm{~cm}^{-1}(-\mathrm{N}=\mathrm{CH})$. NMR: ${ }^{1} \mathrm{H}$ NMR (DMSO-d ${ }_{6}, 300$ $\mathrm{MHz}$ ): $\delta=6.65-7.05(\mathrm{~m}, 7 \mathrm{H}$, aromatic benzene and thiophene), 4.85 (s, $1 \mathrm{H}$, ethylene), 4.95-5.95 (s, $2 \mathrm{H}$, methine), 3.32 (s, $2 \mathrm{H}$, methylene).

M26: 3-[5-(4-chlorophenyl)-5H-[1,3]thiazolo[4,3-b][1,3,4] thiadiazol-2-yl]-2-(thiophen-2-yl)-1,3-thiazolidin-4-one

Color: Brown, Yield: $70 \%$, m. p.: $210-212^{\circ} \mathrm{C}$, MASS: $[\mathrm{M}+1]+$ 437.99, IR: $1665 \mathrm{~cm}^{-1}$ (C=O Str.), $1545 \mathrm{~cm}^{-1}$ (C=C), $2530 \mathrm{~cm}^{-1}$ (Ar. CH), $1540 \mathrm{~cm}^{-1}(-\mathrm{N}=\mathrm{CH})$. NMR: ${ }^{1} \mathrm{H}$ NMR (DMSO-d ${ }_{6}, 300$ $\mathrm{MHz}$ ): $\delta=6.60-7.25(\mathrm{~m}, 7 \mathrm{H}$, aromatic benzene and thiophene), 4.82 (s, $1 \mathrm{H}$, ethylene), 4.90-5.92 (s, $2 \mathrm{H}$, methine), 3.35 (s, $2 \mathrm{H}$, methylene).

R6: 3-[5-(3-chlorophenyl)-5H-[1,3]thiazolo[4,3-b][1,3,4] thiadiazol-2-yl]-2-(3,4dimethoxyphenyl)-1,3-thiazolidin-4-one

Color: Brown, Yield: 85\%, m. p.: 226-228 ${ }^{\circ} \mathrm{C}$, MASS: [M+1] + 492.02, IR: $1675 \mathrm{~cm}^{-1}$ (C=O Str.), $1553 \mathrm{~cm}^{-1}(\mathrm{C}=\mathrm{C}), 2542 \mathrm{~cm}^{-1}$ (Ar. CH), 1580 $\mathrm{cm}^{-1}\left(-\mathrm{N}=\mathrm{CH}\right.$ ). NMR: ${ }^{1} \mathrm{H}$ NMR (DMSO- $d_{6}, 300 \mathrm{MHz}$ ): $\delta=6.50-7.15(\mathrm{~m}$, $7 \mathrm{H}$, aromatic benzene), 4.82 (s, $1 \mathrm{H}$, ethylene), 4.92-5.85 (s, $2 \mathrm{H}$, methine), 3.33 (s, $2 \mathrm{H}$, methylene), 3.75 (s, $6 \mathrm{H}$, methoxy).
W13: 2-(4-chlorophenyl)-3-[5-(3-methoxyphenyl)-5H-[1,3] thiazolo[4,3-b][1,3,4]thiadiazol-2-yl]-1,3-thiazolidin-4-one

Color: White, Yield: $78 \%$, m. p.: $170-172^{\circ} \mathrm{C}$, MASS: $[\mathrm{M}+1]+$ 462.00, IR: $1600 \mathrm{~cm}^{-1}$ (C=O Str.), $1532 \mathrm{~cm}^{-1}$ (C=C), $2520 \mathrm{~cm}^{-1}$ (Ar. $\mathrm{CH}), 1560 \mathrm{~cm}^{-1}(-\mathrm{N}=\mathrm{CH})$. NMR: ${ }^{1} \mathrm{H}$ NMR (DMSO-d, $300 \mathrm{MHz}$ ): $\delta=6.55-7.20(\mathrm{~m}, 8 \mathrm{H}$, aromatic benzene), 4.85 ( $\mathrm{s}, 1 \mathrm{H}$, ethylene), 4.92-5.88 (s, $2 \mathrm{H}$, methine), 3.30 (s, $2 \mathrm{H}$, methylene), 3.78 (s, $3 \mathrm{H}$, methoxy).

W8: 2-(4-fluorophenyl)-3-[5-(3-methoxyphenyl)-5H-[1,3] thiazolo[4,3-b][1,3,4]thiadiazol-2-yl]-1,3-thiazolidin-4-one

Color: Brown, Yield: $80 \%$, m. p.: $160-162^{\circ} \mathrm{C}$, MASS: $[\mathrm{M}+1]+$ 445.55 IR: $1750 \mathrm{~cm}^{-1}$ (C=O Str.), $1682 \mathrm{~cm}^{-1}(\mathrm{C}=\mathrm{C}), 2540 \mathrm{~cm}^{-1}$ (Ar. $\mathrm{CH}), 1670 \mathrm{~cm}^{-1}(-\mathrm{N}=\mathrm{CH})$. NMR: ${ }^{1} \mathrm{H}$ NMR (DMSO-d ${ }_{6}, 300 \mathrm{MHz}$ ): $\delta=6.65-7.05(\mathrm{~m}, 8 \mathrm{H}$, aromatic benzene), 4.80 (s, $1 \mathrm{H}$, ethylene), 4.85-5.90 (s, 2H, methine), 3.34 (s, $2 \mathrm{H}$, methylene), 3.65 (s, $3 \mathrm{H}$, methoxy).

\section{Antitubercular activity}

The synthesized molecules were evaluated for antimycobacterial activity by culturing Mycobacterium smegmatis (NCIM 5138) on Middlebrook $7 \mathrm{H} 9$ broth (Difco) containing 0.5\% albumin, $0.085 \%$ $\mathrm{NaCl}, 0.2 \%$ glucose, $0.05 \%$ Tween 80 , and $0.5 \%$ glycerol at $37^{\circ} \mathrm{C}$ for $48 \mathrm{~h}$ to mid-log phase [optical density at $600 \mathrm{~nm}\left(O D_{600}\right)=$ 0.5]. Minimum inhibitory concentrations (MICs) of antibiotics against $M$. smegmatis were determined in Middlebrook $7 \mathrm{H} 9$ broth by the standard microdilution method. All synthesized derivatives were dissolved in dimethyl sulfoxide and utilized for the antimycobacterial assay in the concentration range of 3.25$1000 \mu \mathrm{g} / \mathrm{mL}$.

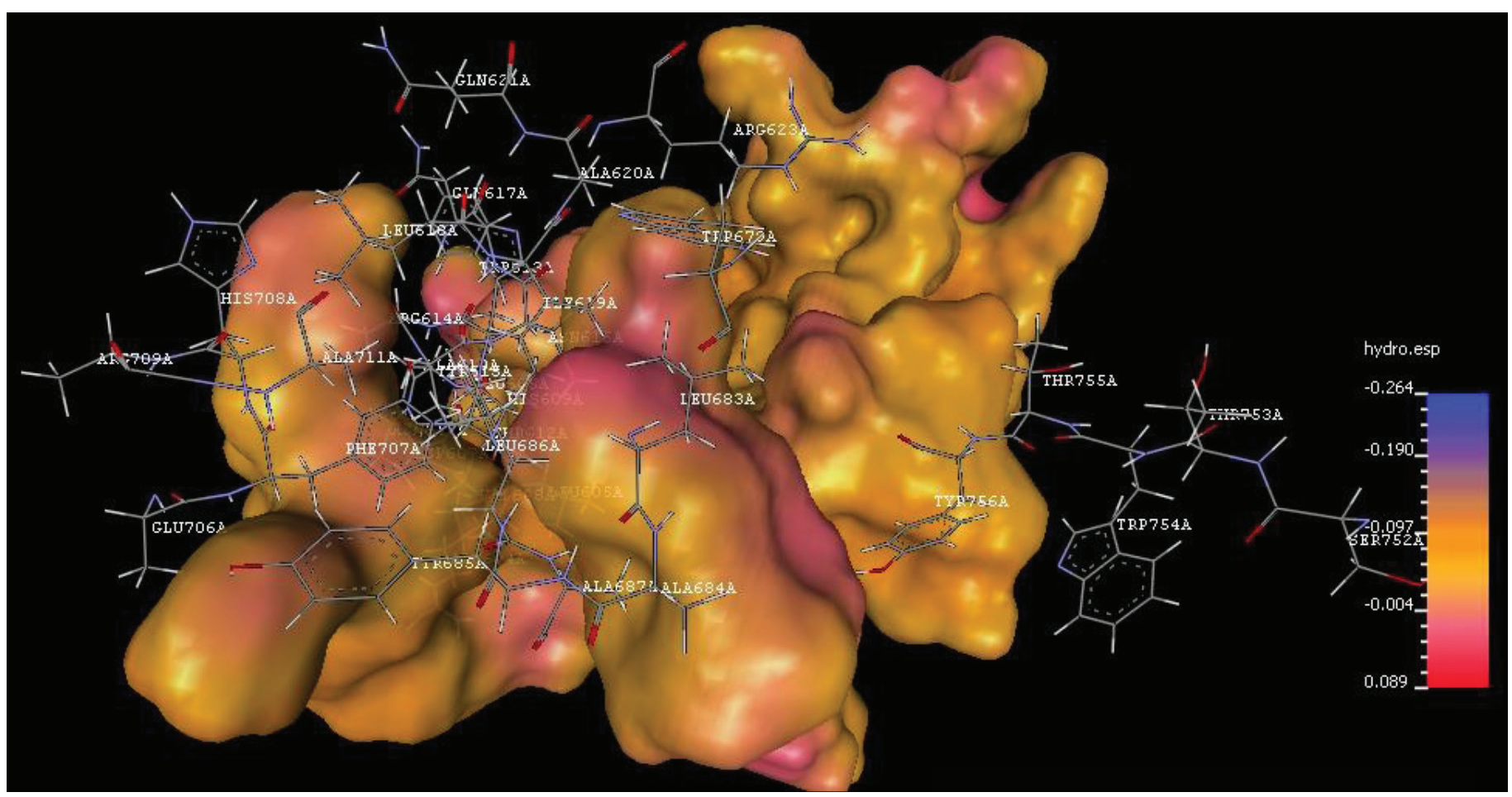

Figure 2. Hydrophobic map of active site of secondary translocase pathway of Mycobacterium tuberculosis 


\section{RESULTS AND DISCUSSION}

\section{Pocket modeling of SEC of M. tuberculosis}

Binding pocket analysis was performed on the $\mathrm{X}$-ray structure of the SEC from M. tuberculosis downloaded from free protein database www.rcsb.org. The ProViz module of Vlife MDS 4.4 was utilized to perform the pocket analysis. An electrostatic and hydrophobic map of the binding pocket of the SEC was generated to identify the relative orientation of critical amino acids. Pocket modeling of the SEC revealed its binding pocket is highly hydrophobic and ASP224, HIS534, and LYS115 are the three important amino acids required for ATP binding. Figure 2 shows the hydrophobic map of the active site of the SEC of $M$. tuberculosis. The SEC runs protein export, which is driven by ATP as the energy source, and inhibition of this ATP binding or blocking three amino acids will inhibit the SEC and ultimately protein export. The binding pocket of the SEC was found to be highly hydrophobic and U-shaped. Molecules were designed with the intent that they will retain necessary hydrophobicity and relative conformation with respect to the SEC of $M$. tuberculosis. Molecules were developed keeping thiazole as the template. Thiazole derivatives due the presence of two heteroatoms that will act as an anchor and two aromatic rings that will act as wings to the nucleus are capable of achieving the bioactive $\mathrm{U}$ - or $\mathrm{V}$-shaped conformation, which is complementary to the binding site of the SEC. Aromatic benzaldehydes are utilized to manipulate the required hydrophobic characters from aromatic interaction with Histidine 534.

\section{Screening based on the drug-like properties}

Pharmacokinetic properties of the molecules are an important factor in the conversion of any New Chemical Entity to the drug. Pre-assessment of these drug-like properties plays a vital role in the selection of potential drug-like candidates from the designed data set of the molecules. All the molecules in the designed set of thiazole derivatives were assessed for Lipinski parameters like molecular weight, $\mathrm{H}$-bond acceptor, $\mathrm{H}$-bond donor, rotatable bond logp, and predicted oral absorption. In all,

\section{Table 3. Table showing the molecules having the desired drug-like properties}

\begin{tabular}{|c|c|c|c|c|c|c|c|c|}
\hline Sr. no. & Compound code & Mole. wt. & $\mathrm{H}$-acce & H-donor count & RBC & $\log P$ & TPSA (Å) & Pre. $\%$ oral abs. \\
\hline 1 & A10 & 476.988 & 4 & 0 & 4 & 4.738 & 44.81 & 93.54 \\
\hline 2 & A11 & 476.988 & 4 & 0 & 4 & 4.738 & 81.4 & 80.91 \\
\hline 3 & B3 & 476.442 & 1 & 0 & 3 & 4.939 & 35.57 & 96.72 \\
\hline 4 & $\mathrm{C} 1$ & 431.99 & 1 & 0 & 3 & 4.83 & 35.57 & 96.72 \\
\hline 5 & $\mathrm{C} 13$ & 431.99 & 1 & 0 & 3 & 4.83 & 35.57 & 96.72 \\
\hline 6 & F1 & 492.043 & 3 & 0 & 7 & 4.847 & 54.04 & 90.35 \\
\hline 7 & $\mathrm{H} 25$ & 405.498 & 2 & 0 & 3 & 3.908 & 46.57 & 85.26 \\
\hline 8 & L13 & 475.059 & 2 & 0 & 6 & 4.896 & 41.37 & 94.72 \\
\hline 9 & M9 & 447.99 & 2 & 1 & 4 & 4.535 & 58.63 & 88.77 \\
\hline 10 & M26 & 438.019 & 1 & 0 & 3 & 4.891 & 35.57 & 96.72 \\
\hline 11 & 013 & 447.99 & 2 & 1 & 4 & 4.535 & 58.63 & 88.77 \\
\hline 12 & 017 & 447.99 & 2 & 1 & 4 & 4.535 & 58.63 & 88.77 \\
\hline 13 & Q21 & 447.56 & 3 & 1 & 7 & 3.873 & 81 & 81.05 \\
\hline 14 & Q22 & 417.534 & 2 & 0 & 5 & 3.778 & 57.95 & 89.00 \\
\hline 15 & Q24 & 433.533 & 3 & 1 & 6 & 3.483 & 81 & 81.05 \\
\hline 16 & Q30 & 431.517 & 1 & 0 & 3 & 3.498 & 67.18 & 85.82 \\
\hline 17 & R6 & 492.043 & 3 & 0 & 7 & 4.847 & 54.04 & 90.35 \\
\hline 18 & R25 & 421.952 & 1 & 0 & 3 & 4.423 & 48.71 & 92.19505 \\
\hline 19 & $\mathrm{R} 26$ & 438.019 & 1 & 0 & 3 & 4.891 & 35.57 & 96.72 \\
\hline 20 & U8 & 491.588 & 5 & 1 & 8 & 4.038 & 77.1 & 82.40 \\
\hline 21 & V3 & 457.598 & 3 & 1 & 7 & 4.28 & 67.86 & 85.58 \\
\hline 22 & w8 & 445.562 & 3 & 0 & 5 & 4.324 & 44.81 & 93.54 \\
\hline 23 & W13 & 462.017 & 2 & 0 & 5 & 4.838 & 44.81 & 93.54 \\
\hline
\end{tabular}

TPSA: Total polar surface area, RBC: Red blood cell 


\begin{tabular}{|c|c|c|c|c|}
\hline Sr. no. & Compound code & Binding energy & $\mathrm{H}$-bond & Pi-stacking \\
\hline 1. & A10 & -33.43 & HIS534 & HIS534 \\
\hline 2. & A11 & -0.12 & HIS534 & HIS534 \\
\hline 3. & B3 & -35.40 & HIS534 & HIS534 \\
\hline 4. & $\mathrm{C} 1$ & -7.41 & ASP224 & HIS534 \\
\hline 5. & $\mathrm{C} 13$ & -2.31 & $\begin{array}{l}\text { HIS534 } \\
\text { HIS534 }\end{array}$ & HIS534 \\
\hline 6. & F1 & -33.32 & HIS534 & HIS534 \\
\hline 7. & L13 & -8.74 & HIS534 & HIS534 \\
\hline 8. & M9 & -37.54 & ASP219 & HIS534 \\
\hline 9. & M26 & -38.54 & ASP224 & HIS534 \\
\hline 10. & 013 & -11.91 & GLN498 & HIS534 \\
\hline 11. & 017 & -9.81 & HIS534 & HIS534 \\
\hline 12. & Q21 & 23.92 & ASP219 & HIS534 \\
\hline 13. & Q22 & -11.97 & HIS534 & HIS534 \\
\hline 14. & Q24 & -11.03 & HIS534 & HIS534 \\
\hline 15. & Q30 & -3.89 & HIS534 & HIS534 \\
\hline 16. & R25 & -2.51 & HIS534 & HIS534 \\
\hline 17. & R26 & -4.66 & ASP224 & HIS534 \\
\hline 18. & R6 & -11.74 & HIS534 & HIS534 \\
\hline 19. & U8 & -0.21 & HIS534 & HIS534 \\
\hline 20. & V3 & -36.40 & HIS534 & HIS534 \\
\hline 21. & $\mathrm{H} 25$ & -8.37 & HIS534 & HIS534 \\
\hline 22. & W8 & -33.40 & ASP224 & HIS534 \\
\hline 23. & W13 & -31.56 & HIS534 & HIS534 \\
\hline
\end{tabular}

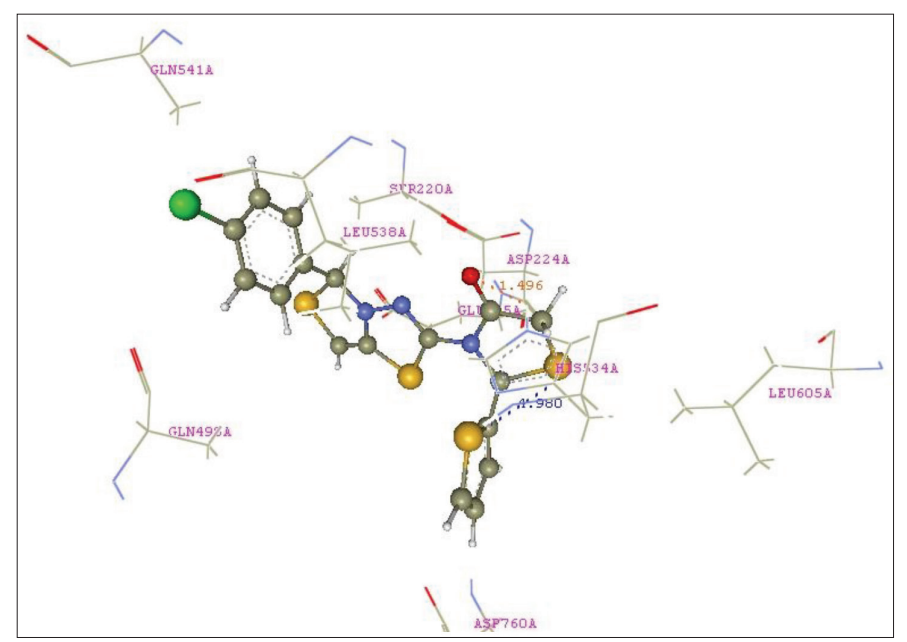

Figure 3. Figure showing posed molecule M26 (Ball and Stick) with PDB ID $4 \cup A Q$ with hydrogen bond interaction (red color) and Pi-Stacking Interaction (blue colour)

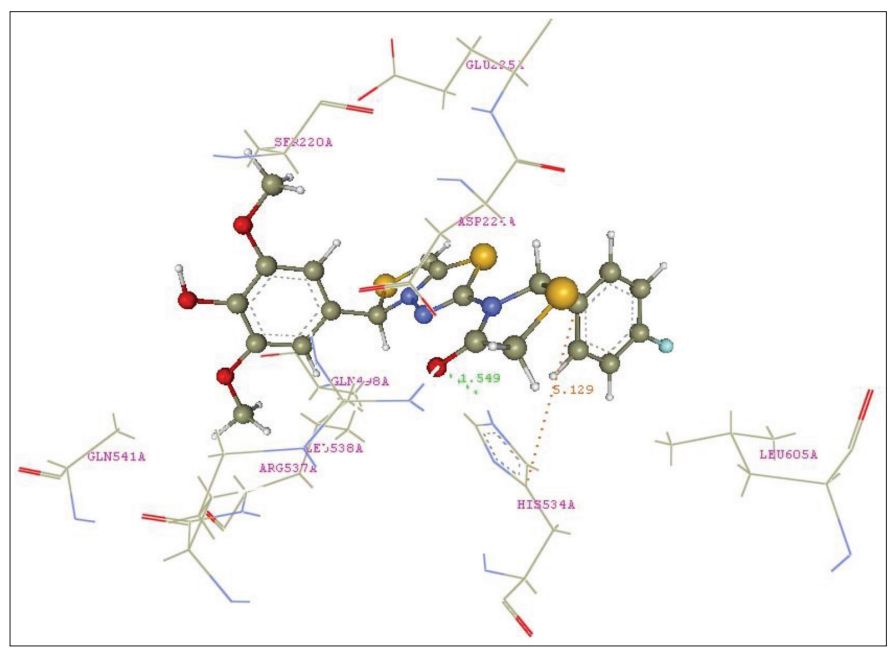

Figure 4. Showing the posed molecule U8 (Ball and Stick) with PDB ID $\triangle \cup A Q$ with hydrogen bond interaction (red color)

23 derivatives were found to have the desired pharmacokinetic properties and predicted oral absorption $<70 \%$. The selected 23 derivatives with the drug-like properties are given in Table 3.

\section{Molecular docking}

Molecular docking was utilized to predict the potential active inhibitors from the designed set of ligands. Grip-based docking analysis was performed maintaining the SEC in rigid conformation and ligands in flexible conformation. All the design set of molecules were found to be binding to the same binding site to that of ATP in the SEC. The designed set of molecules was found to be interacting with HIS 534 and ASP224 via formation with hydrogen bond interaction and pi-stacking interaction. Binding interactions of the 23 selected molecules are summarized in Table 4, while Figures 3, 4, 5, and 6 show the most active conformation of molecules M26, U8, R26, and A10 (Ball and Stick) with PDB ID 4UAQ, respectively.

\section{Biological activity}

The antimycobacterial activity of the synthesized derivatives was determined against the standard M. smegmatis (NCIM No: 5138). M. smegmatis is an organism belonging to the family Mycobacterium having nearly $80 \%$ genome similarity with $M$. tuberculosis. Isoniazid was utilized as the positive standard for the antimycobacterial activity. MICs of all 23 derivatives are summarized in Figure 7. All the compounds showed good to moderate activity against the tested strain. Compounds R26, Q30, and M9 showed maximum antitubercular activity (MIC: $62.5 \mu \mathrm{g} / \mathrm{mL}$ ). The excellent activity of M9 and R26 indicates halogens have a positive effect on antitubercular activity, and the activity of Q30 is an interesting finding that indicates substitution of the heterocyclic nucleus will also potentiate the activity. Derivative L13 showed good activity (MIC: $125 \mu \mathrm{g} / \mathrm{mL}$ ), which justifies the substitution of halogen in the aromatic ring. A10, C13, F1, O13, O17, Q21, Q22, Q24, R25, R6, U8, and W13 are moderately active compounds with MIC: $500 \mu \mathrm{g} / \mathrm{mL}$. 


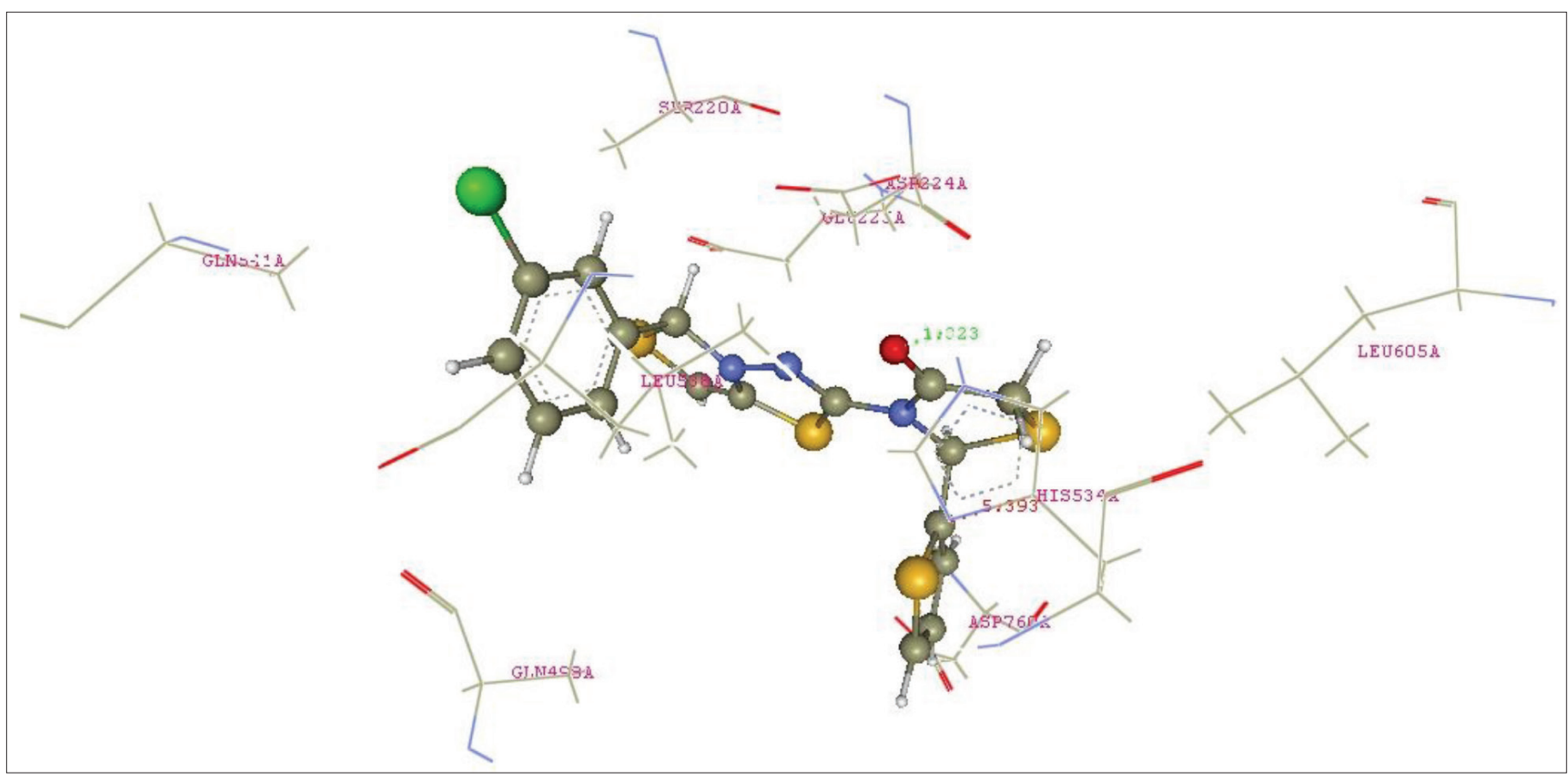

Figure 5. Showing the posed molecule R26 (Ball and Stick) with PDB ID 4UAQ with hydrogen bond interaction (red color)

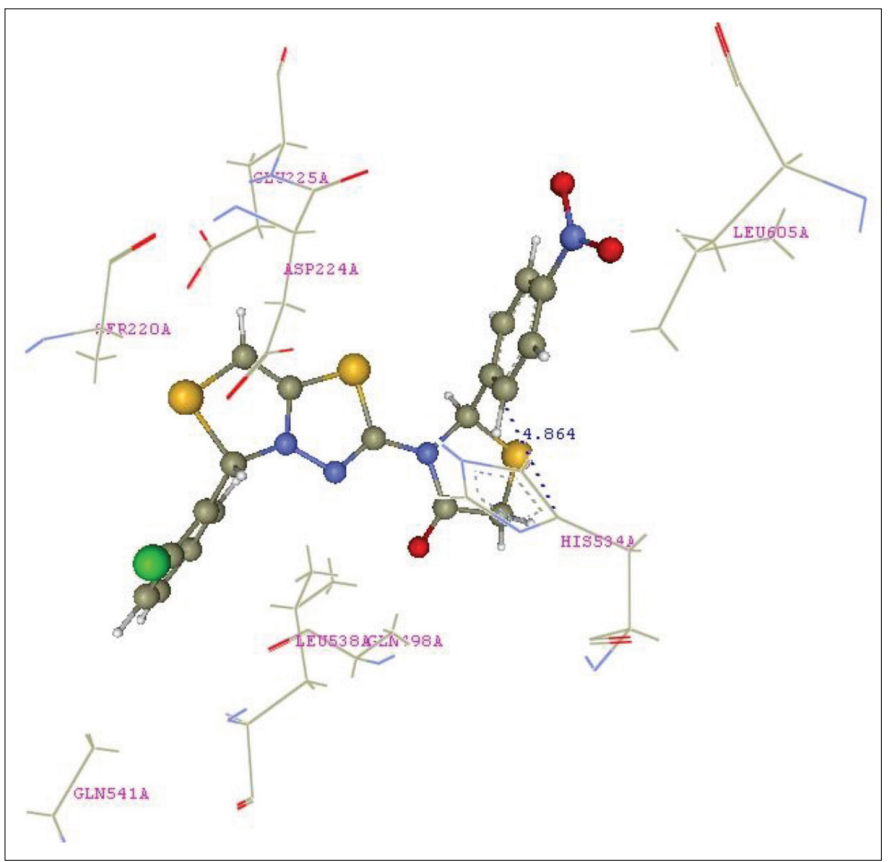

Figure 6. Showing the posed molecule A10 (Ball and Stick) with PDB ID $4 \cup A Q$

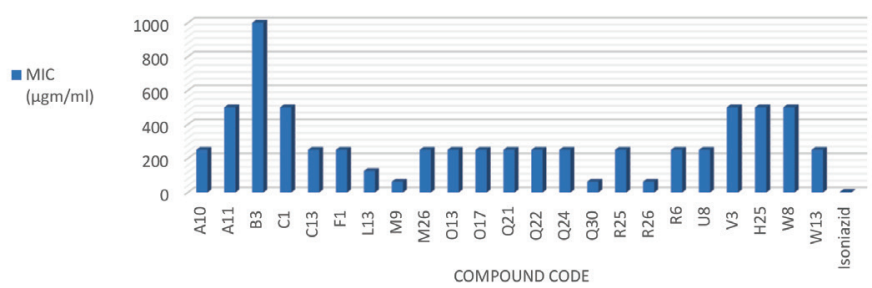

Figure 7. Designed compounds with minimum inhibitory concentration

\section{CONCLUSIONS}

Attempts to design and develop molecules targeting secretory systems of $M$. tuberculosis yielded the following significant findings. Identification and validation of secretory systems as targets for antitubercular drug design and discovery were successfully carried out. Out of the three protein export systems associated with $M$. tuberculosis the SEC protein export system was utilized successfully for the development of antitubercular agents that are selective and can be utilized against all resistant forms of tuberculosis. Protein export systems are conserved systems and they have no isoform in humans and so targeting protein export systems will be the potential route for the development of selective and active antitubercular agents. Pocket modeling of the active site or binding site of the SEC revealed an interesting fact about the size and shape of the binding pocket of the SEC and signified the development of hydrophobic ligands for binding with the SEC of M. tuberculosis. Based on the pocket modeling data and literature survey in total 5184 molecules were designed around the thiazole scaffold via the change in the different aromatic benzaldehyde structures. Moreover, 5625 designed molecules were further scrutinized via Lipinski drug-like properties and toxicity profile using OCHEM and for binding efficiency using molecular docking analysis. The results of all three analyses yielded 23 potent, selective molecules successfully synthesized via reaction of an aromatic aldehyde, thiosemicarbazide, and thioglycolic acid. All the molecules were successfully synthesized using microwave-assisted synthesis, which improved the yield and reduced the time of reaction compared to the procedures reported in the literature. All 23 derivatives synthesized were characterized via all physicochemical methods, melting point, and IR and NMR spectroscopy. Halogen-substituted derivatives 
showed significant activity, which indicates substitution of an electron-withdrawing group in the aryl ring will potentiate antimycobacterial activity. M26, U8, and R26 molecules have significant desirable biological activity and specific interactions with the SEC. Further optimization of these leads is necessary for the development of potential antitubercular drug-like candidates. These potential drug candidates with specific SEC inhibitory properties resulted from the utilization of integration of pocket modeling and virtual screening.

Conflict of Interest: No conflict of interest was declared by the authors.

\section{REFERENCES}

1. Abagyan R, Totrov M, High-throughput Docking for Lead Generation. Curr Opin Chem Biol. 2001;5:375-382.

2. Alam S, Khan F. QSAR and Docking studies on Xanthone Derivatives for Anticancer Activity Targeting DNA topoisomerase Il $\alpha$. Drug Des Devel Ther. 2014;8:183-195.

3. Bermudez LE, Goodman J. Mycobacterium tuberculosis invades and replicates within Type II alveolar cells. Infect Immun. 1996;64: 14001406.

4. Bhatia MS, Ingale KB, Choudhari PB, Bhatia NM, Sawant RL. Application quantum and physicochemical molecular descriptors utilizing principal components to study the mode of anticoagulant activity of pyridyl chromen-2-one derivatives. Bioorg Med Chem. 2009;17:1654-1662.

5. Borrell S, Gagneux S. Infectiousness, reproductive fitness, and evolution of drug-resistant Mycobacterium tuberculosis. Int J Tuberc Lung Dis. 2009;13:1456-1466.

6. Brodin P, Majlessi L, Marsollier L, de Jonge MI, Bottai D, Demangel C, Hinds J, Neyrolles O, Butcher PD, Leclerc C, Cole ST, Brosch R. Dissection of ESAT-6 system of Mycobacterium tuberculosis and impact on immunogenicity and virulence. Infect Immun. 2006;74:88-98.

7. Choudhari PB, Bhatia MS, Bhatia NM. Application of Pocket Modeling and k-nearest Neighbor Molecular Field Analysis (kNN-MFA) for Designing of some Anticoagulants: Potential Factor IXa Inhibitors. Medicinal Chemistry Research. 2012;19:3-5.

8. Choure R, Pitre KS. Structural modification of coumarin for its increased anticoagulation potency. Canadian J Chem Engineering Technology. 2010;1:7-15.

9. Derrick SC, Morris SL. The ESAT6 protein of Mycobacterium tuberculosis induces apoptosis of macrophages by activating caspase expression. Cell Microbiol. 2007;9:1547-1555.
10. Ducati RG, Ruffino-Netto A, Basso LA, Santos DS. The Resumption of Consumption - A Review on Tuberculosis. Mem Inst Oswaldo Cruz. 2006;101:697-714.

11. Feltcher ME, Sullivan JT, Braunstein M. Protein Export Systems of Mycobacterium tuberculosis: novel target for drug development. Future Microbiol. 2010;5:1581-1597.

12. Fenton MJ, Vermeulen MW. Immunopathology of tuberculosis: roles of macrophages and monocytes. Infect Immun. 1996;64:683-690.

13. Finlay BB, Falkow S. Common themes in microbial pathogenicity revisited. Microbiol Mol Biol Rev. 1997;61:136-169.

14. Fortune SM, Jaeger A, Sarracino DA, Chase MR, Sassetti CM, Sherman $\mathrm{DR}$, Bloom BR, Rubin EJ. Mutually dependent secretion of proteins required for mycobacterial virulence. Proc Natl Acad Sci USA. 2005;102:10676-10681.

15. Ginsberg AM. Tuberculosis drug development: Progress challenges and the road ahead. Tuberculosis (Edinb). 2010;90:162-167.

16. Jain A, Mondal R. Extensively drug-resistant tuberculosis: current challenges and threats. FEMS Immunol Med Microbiol. 2008;53:145150.

17. Keane J, Balcewicz-Sablinska MK, Remold HG, Chupp GL, Meek $\mathrm{BB}$, Fenton MJ, Kornfeld H. Infection by Mycobacterium tuberculosis promotes human alveolar macrophages apoptosis. Infect Immun. 1997;65:298-304.

18. Leung AN. Pulmonary Tuberculosis: The Essentials. Radiology. 1999;210:307-322.

19. Sugie $\mathrm{Y}$, Inagaki $\mathrm{S}$, Kato $\mathrm{Y}$, Nishida $\mathrm{H}$, Pang $\mathrm{CH}$, Saito $\mathrm{T}$, Sakemi $\mathrm{S}$, Dib-Hajj F, Mueller JP, Sutcliffe J, Kojima Y. CJ-21,058, a new SecA inhibitor isolated from a fungus. J Antibiot (Tokyo). 2002;55:25-29.

20. Jang MY, De Jonghe S, Segers K, Anné J, Herdewijn P. Synthesis of novel 5-amino-thiazolo[4,5-d]pyrimidines as $E$. coli and S. aureus SecA inhibitors. Bioorg Med Chem. 2011;19:702-714.

21. Tripathi L, Kumar P, Singh R, Stables JP. Design, synthesis and anticonvulsant evaluation of novel $\mathrm{N}$-(4-substituted phenyl)-2-[4(substituted) benzylidene]-hydrazinecarbothioamides. Eur $\mathrm{J}$ Med Chem. 2012;47:153-166.

22. Malipeddi H, Karigar AA, Malipeddi VR, Sikarwar MS. Synthesis and Antitubercular Activity of Some Novel Thiazolidinone Derivatives. TroJ of Pharm Res. 2012;11:611-620. 